\title{
INCREASING THE EFFICIENCY OF MULTY-VARIANT CALCULATIONS OF ELECTROMAGNETIC FIELD DISTRIBUTION IN MATRIX OF A POLYGRADIENT SEPARATOR
}

\author{
Jasim Mohmed Jasim Jasim \\ Department of Electrical Power Engineering Techniques \\ Al-Furat Al-Awsat Technical University - Al-Musssaib Technical College \\ 15 Al-Hilla-Najaf main road, Al-Kufa, Iraq, 54001 \\ Iryna Shvedchykova $\bowtie$ \\ Department of Computer Engineering and Electromechanics ${ }^{1}$ \\ shvedchykova.io@knutd.edu.ua \\ Igor Panasiuk \\ Department of Heat Power Engineering, Resource Saving \\ and Technogenic Safety ${ }^{l}$ \\ Julia Romanchenko \\ Department of Electrical Engineering ${ }^{2}$ \\ Inna Melkonova \\ Department of Electrical Engineering ${ }^{2}$ \\ ${ }^{1}$ Kyiv National University of Technologies and Design \\ 2 Nemyrovycha-Danchenka str., Kyiv, Ukraine, 01011 \\ ${ }^{2}$ Volodymyr Dahl East Ukrainian National University \\ 59-a Tsentralnyi ave., Severodonetsk, Ukraine, 93400
}

Corresponding author

\begin{abstract}
An approach is proposed to carry out multivariate calculations of the magnetic field distribution in the working gaps of a plate polygradient matrix of an electromagnetic separator, based on a combination of the advantages of two- and three-dimensional computer modeling. Two-dimensional geometric models of computational domains are developed, which differ in the geometric dimensions of the plate matrix elements and working air gaps. To determine the vector magnetic potential at the boundaries of two-dimensional computational domains, a computational 3D experiment is carried out. For this, three variants of the electromagnetic separator are selected, which differ in the size of the working air gaps of the polygradient matrices. For them, three-dimensional computer models are built, the spatial distribution of the magnetic field in the working intervals of the electromagnetic separator matrix and the obtained numerical values of the vector magnetic potential at the boundaries of the computational domains are investigated. The determination of the values of the vector magnetic potential for all other models is carried out by interpolation. The obtained values of the vector magnetic potential are used to set the boundary conditions in a computational 2D experiment. An approach to the choice of a rational version of a lamellar matrix is substantiated, which provides a solution to the problem according to the criterion of the effective area of the working area. Using the method of simple enumeration, a variant of the structure of a polygradient matrix with rational geometric parameters is selected. The productivity of the electromagnetic separator with rational geometric parameters of the matrix increased by $3-5 \%$ with the same efficiency of extraction of ferromagnetic inclusions in comparison with the basic version of the device.
\end{abstract}

Keywords: electromagnetic separator, electromagnetic field, boundary conditions, vector magnetic potential, finite element method. 


\section{Introduction}

The most effective technology for extracting ferromagnetic particles up to $1 \mathrm{~mm}$ in size from various materials is polygradient magnetic separation. The working body of polygradient electromagnetic separators is a stationary matrix or a rotating rotor with a polygradient medium made of ferromagnetic bodies (balls, cylinders, rods, steel wool, pointed plates, etc.).

Most of the existing devices for polygradient magnetic separation are designed for purification of liquid media (pulp) [1]. For wet magnetic separation of weakly magnetic materials, rotary electromagnetic Jones separators of KHD Humboldt Wedag (Germany) with working ferromagnetic bodies in the form of corrugated plates are widely used; electromagnetic separators made by Sala (Sweden) and Metso minerals (Finland), which use steel mesh or cotton wool. Such devices are distinguished by a high efficiency of the working process, ensuring the removal of almost all highly magnetic particles from the pulp [2].

In connection with the growing requirements for product quality, the problem of removing finely dispersed ferromagnetic inclusions arising in the process of equipment wear is also relevant for bulk materials (flour, grain, sugar, medicinal mixtures, kaolin, etc.). The use of the above types of electromagnetic separators for solving the problems of cleaning bulk materials is economically inexpedient due to their high cost, metal and energy consumption, and the presence of small working air gaps. For cleaning bulk materials, open multi-pole magnetic systems are used, which are insufficiently effective in removing ferromagnetic impurities up to $1 \mathrm{~mm}$ in size. Therefore, reliable and effective cleaning of bulk materials from small metal impurities can only be provided by devices specially developed for these purposes by numerous manufacturers of magnetoseparation devices (for example, Ukrprommagnit (Ukraine), Erieze (USA), CALAMIT (Germany)). This article discusses a non-contact polygradient electromagnetic separator for cleaning bulk materials with a lamellar matrix, developed with the participation of the authors of the work. The basic version of the separator was installed at the Druzhkovka porcelain factory (Ukraine) to control the quality of kaolin purification. The device shows a fairly high efficiency of extraction of ferromagnetic inclusions, which amounted to $90.5-95.5 \%$.

An important stage in the design of polygradient electromagnetic separators designed to extract ferromagnetic particles up to $1 \mathrm{~mm}$ in size from dispersed media is the procedure for determining the rational geometric parameters of their plate matrices, which is multivariate. Usually, numerical methods for calculating magnetic fields and specialized software for two or three-dimensional modeling are used to solve it. The use of two-dimensional models is based on certain assumptions regarding the boundary conditions and the nature of the distribution of the magnetic field in the working intervals and does not require significant computing resources and calculation time. The advantages of three-dimensional models are taking into account the real geometrical dimensions of magnetic systems and the possibility of studying the influence of geometry on the flux distribution in any element of the magnetic circuit. At the same time, the determination of the spatial distribution of the field in complex three-dimensional models of electromagnetic separators is a rather laborious and time-consuming process. This determines the urgency of developing new approaches to multivariate calculations of magnetic fields in polygradient matrices of electromagnetic separators capable of providing the necessary computational efficiency and accuracy.

Numerous publications are devoted to the study of the distribution of the magnetic field in the working body (matrix) of polygradient magnetic separators. In work [3] it is shown that the method of polygradient magnetic separation is effective for removing or filtering small and weakly magnetic particles and is widely used for processing minerals, water purification, purification of cells and proteins. It is noted that the magnetic matrix is the most important structural element of the magnetic separator. The material, geometry, dimensions and arrangement of matrix elements can significantly affect the gradient and distribution of the magnetic field. But questions about the influence of the structural elements of the matrix on the performance of the separator remained unresolved. In [4], a new design of the rod matrix is described, which provides higher values of the magnetic field gradient. To study the influence of the structural parameters of the matrix on the efficiency of extraction of fine particles, a numerical-field analysis of the spatial distribution of the magnetic field by the finite element method (FEM) is carried out using the software Ansoft 
Maxwell 3D (USA). The tetrahedron is adopted as the main mesh element in the modeling. For each model, about 100,000 tetrahedrons are used. The average time spent on simulation is about 6-8 hours in a personal computer with a Pentium 4 processor. Due to the high complexity of calculations, a limited number of standard sizes of matrix rods with a diameter of 3.0 and $3.5 \mathrm{~mm}$ are investigated. Taking this into account, in [5], an approach is proposed for calculating the magnetostatic field in a doubly periodic heterogeneous medium (separator matrix) by the method of integral equations. The integral equation is formulated for the magnetization vector of the elements of the medium. The calculation of the field characteristics is carried out by solving the field problem in the area of the main parallelogram of periods without setting boundary conditions on its sides. Calculated expressions for the field strength and magnetic permeability tensor are obtained. The results of the study of the magnetic force field of the matrix of high-gradient magnetic separator are presented. At the same time, the method proposed in [5], although it is universal, is developed for calculating the characteristics of the magnetic field in local zones.

As the analysis of publications [6-11] shows, a more flexible FEM is mainly used for calculating the magnetic fields of electromagnetic devices. Thus, in [6], to study the effect of the configuration of the elements of the separator matrix on the efficiency of particle capture in high-gradient magnetic fields, three-dimensional finite element models were analyzed using the COMSOL Multiphysics software product (Sweden). The research results presented in [7], obtained only for a certain configuration of the polygradient matrix medium based on a single and multielement magnetic wire. It remains unclear whether these results can be extended to other types of polygradient media.

In [8], the principles of automated formation of two-dimensional geometric models of electrical machines for calculating their magnetic fields using the FEMM program (USA) are shown. The article [9] presents the principles and results of the numerical-field determination of the electromagnetic and energy parameters of three-phase asynchronous motors for the purpose of their verification analysis also using the FEMM program (USA). The calculation results obtained in a two-dimensional formulation require further experimental verification.

The article [10] reflects the results of numerous studies of two types of permanent magnet electric generators. The calculation of the characteristics of the generators under study was carried out in a three-dimensional setting using the Simcenter MagNet and Simcenter MotorSolve software packages. The article [11] presents the results of research on the performance characteristics of a permanent magnet disk generator. The calculation of the magnetic field and device characteristics was carried out using the Infolytica Magnet software package. The expediency of carrying out calculations using three-dimensional computer models has been substantiated, but their high labor intensity has been noted.

Thus, the analysis of publications allows to assert that in the study of magnetic fields of electromagnetic devices, in particular magnetic separators, numerical methods have found wide application, first of all, FEM in a two- or three-dimensional formulation. The experience of using numerical methods in calculating magnetic fields has shown that three-dimensional modeling requires significant computational and time resources, and the use of two-dimensional models is associated with certain assumptions. Therefore, it is expedient to conduct a study devoted to the development of a more efficient and universal combined approach to the analysis of magnetic fields, taking into account the advantages of finite element analysis in both two-dimensional and three-dimensional formulations.

The aim of research is the development and practical implementation of an approach that allows for multivariate calculations of the magnetic field distribution in the working gaps of the plate matrix of the electromagnetic separator with increased computational efficiency.

To achieve the aim, the following objectives are set:

- to substantiate an approach to carrying out multivariate calculations of the magnetic field distribution;

- to determine the boundary conditions for the working areas of the matrix using the selected three-dimensional models of the electromagnetic separator;

- to carry out variant calculations and determine the rational geometric parameters of the plate elements of the magnetic matrix using two-dimensional models of working zones. 


\section{Substantiation of the approach to carrying out multivariate calculations of the magnetic field distribution}

The previous author's works [12-14] reflect the results of studies of a polygradient electromagnetic separator designed for cleaning bulk materials from fine ferromagnetic impurities up to $1 \mathrm{~mm}$ in size, namely:

- using the methodological tools of genetic synthesis and symmetry transformations, six structural variants of the lamellar polygradient matrix of an electromagnetic separator based on triangular elements are obtained [12];

- a preliminary comparative analysis of the synthesized structures is carried out to assess the degree of inhomogeneity of the magnetic field in their working intervals using a numerical MSE in a two-dimensional formulation [13];

- a rational structural version of the plate matrix of the separator is substantiated, the geometric model of which, indicating the main geometric dimensions, is shown in Fig. 1 [14].
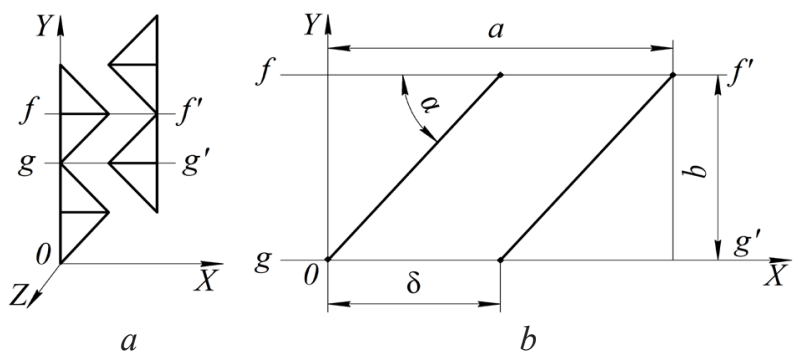

Fig. 1. Rational structural version of the lamellar matrix:

$a$-geometric model; $b$ - the main geometric dimensions of the working area

The main geometrical dimensions of the working area (Fig. 1): $\delta$ - pole-to-pole working gap; $\alpha$ - angle at the top of the pole protrusion; $a$-working width of the matrix; $b$ is the base of the pole performance. The following geometric similarity criteria were set for the regions under study: $X_{1}=b / a$ and $X_{2}=\alpha$. The ranges of variation of the geometric similarity criteria $X_{1}$ and $X_{2}$, which are of practical interest, were: $X_{1}=b / a=0.18 \ldots 0.4, X_{2}=\alpha=0.11 \pi \ldots 0.18 \pi$. The thickness $t$ of the matrix plates (not shown in Fig. 1) in the direction of the $0 \mathrm{z}$ axis was taken $t=6 \mathrm{~mm}$. Taking into account the accepted ranges of variation of the geometric similarity criteria, 55 variants of the polygradient matrix were obtained and 2D models of their working clearances were constructed (Fig. 2), for which the boundary conditions were required to be specified. In particular, it was necessary to determine the values of the vector magnetic potential along the boundaries $c h$ and $d e$ in Fig. 2, which coincide with the lines of force of the computational domain. In this case, if for the vector magnetic potential along one of the boundaries (for example, for the boundary $c h$ ), the zero value $A_{c h}=0 \mathrm{can}$ be taken, then the vector magnetic potential $A_{d e}$ along the second boundary must be determined.

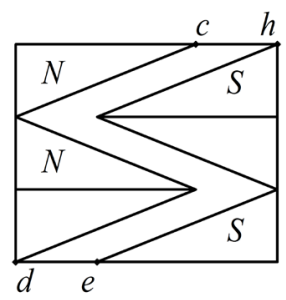

Fig. 2. 2D-model of the working area

The difference in the values of the vector magnetic potential $\left|\overline{A_{d e}}-\overline{A_{c h}}\right|=\left|\overline{A_{d e}}\right|$ is equal to the average flux of the magnetic induction vector $\Phi$ in the working air gap, which is per unit of pole thickness $\mathrm{t}$ in the direction of the $0 z$ axis (Fig. 1, $\boldsymbol{a} ; t=6 \mathrm{~mm}$ ). That is, it is determined by the ratio $\left|\overline{A_{d e}}\right|=\Phi / t$. Therefore, to determine the value of the potential $A_{d e}$, it is necessary to calculate the magnetic flux $\Phi$. This problem can be solved only with the use of three-dimensional computer models. 
To increase the computational efficiency of calculations of the magnetic flux and vector magnetic potential, it was decided to limit the construction and study of only three three-dimensional models of the electromagnetic system of the separator, which differ in the size of the working air gap $\delta$. For further analysis, the following three options of the electromagnetic separator were selected:

- option 1 (with the maximum $\delta_{\max }$ value of the working gap): the distance between adjacent projections of the plates $b=32.4 \mathrm{~mm}\left(X_{1}=b / a=0.18\right)$, the angle at the apex of the pole protrusion $2 \alpha=54^{\circ}\left(X_{2}=\alpha=0.15 \pi\right)$, working pole gap $\delta=53 \mathrm{~mm}$;

- option 2 (with an average value $\delta_{a v}$ of the working gap, which satisfies the condition $\left.\delta_{\min } \leq \delta_{a v} \leq \delta_{\max }\right)$ : the distance between adjacent protrusions of the $b=72 \mathrm{~mm}\left(X_{1}=b / a=0,4\right)$, the angle at the apex of the pole protrusion $2 \alpha=66^{\circ}\left(X_{2}=\alpha=0.18 \pi\right)$, working pole gap $\delta=37 \mathrm{~mm}$;

- option 3 (with a minimum $\delta_{\min }$ value of the working gap): the distance between adjacent projections of the plates $b=50 \mathrm{~mm}\left(X_{1}=b / a=0.28\right)$, the angle at the apex of the pole protrusion $2 \alpha=40^{\circ}\left(X_{2}=\alpha=0.11 \pi\right)$, the working pole gap $\delta=13.6 \mathrm{~mm}$. These parameters correspond to the basic design of the electromagnetic separator.

It should be noted that the air gap $\delta$ is determined through the geometric similarity criteria $X_{1}=b / a$ та $X_{2}=\alpha$. For the selected models, the air gap $\delta$ varies in a fairly wide range: from $\delta=13.6 \mathrm{~mm}$ (option 3 ) to $\delta=53 \mathrm{~mm}$ (option 1 ). The determination of the values of the vector magnetic potential for all other models was carried out by interpolation.

To determine the magnetic flux $\Phi$ in the working air gaps, the Magnet module of the Infolytica software package (Canada) was used [15]. The construction of the geometry of the 3D model (Fig. 3) of the electromagnetic separator for the purpose of importing it into the Magnet module was carried out in the KOMPAS program (Russia). Fig. 3 shows on the example of option $1(\delta=53 \mathrm{~mm})$ a three-dimensional geometric model of an electromagnetic separator (Fig. $\mathbf{3}, \boldsymbol{a})$ and a picture of the magnetic field distribution (Fig. 3, b).

When modeling, the following initial data were taken: current strength in the windings $-3.2 \mathrm{~A}$; material of the magnetic circuit, matrix plates, matrix walls, which are parallel to the poles of the magnetic circuit - steel grade St. 3, the other two walls are aluminum.
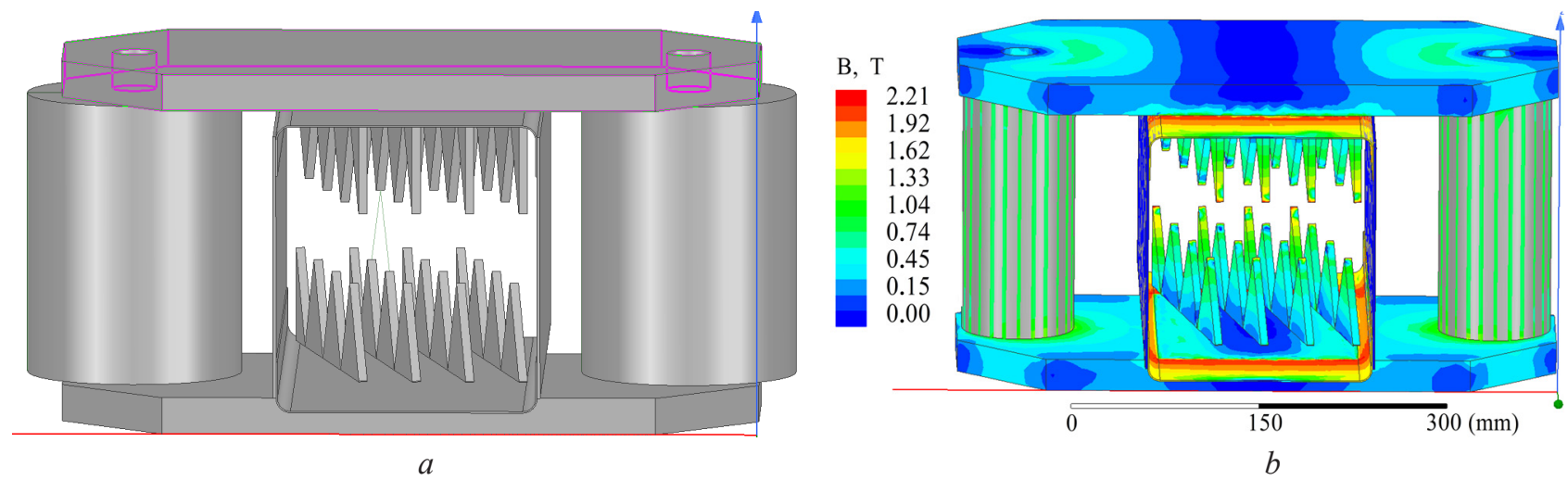

Fig. 3. Three-dimensional modeling of an electromagnetic separator: $a$-geometric model of the separator; $b$ - distribution pattern of the magnetic field

To substantiate the possibility of using the Infolytica software package, a test task was solved and a 3D computer model of the electromagnetic separator was verified. The verification results showed that the use of the Infolytica program for computer modeling allows obtaining quantitative and qualitative data on the distribution of the magnetic field. These data can serve as a basis for further studies of the magnetic flux in the working interpole zone of the electromagnetic separator [16].

\section{The results of research to determine the boundary conditions for the working areas of the matrix}

Calculations of the magnetic flux $\Phi$ through the area $S$ (Fig. 4) with a width of $t=6 \mathrm{~mm}$, limiting the working air gap of the separator matrix, have been carried out. According to these 
calculations, the magnetic flux $\Phi$ through the site $\mathrm{S}$ was: for option $1-0.0000126111 \mathrm{~Wb}$, for option $2-0.0000558067 \mathrm{~Wb}$, for option $3-0.0000640057 \mathrm{~Wb}$. Accordingly, the value of the vector magnetic potential $A_{d e}$ in the section $d e$ (Fig. 2) was: for option $1-0.00630554 \mathrm{~Wb} / \mathrm{m}$, for option $2-0.027903332 \mathrm{~Wb} / \mathrm{m}$, for option $3-0.032002859 \mathrm{~Wb} / \mathrm{m}$.

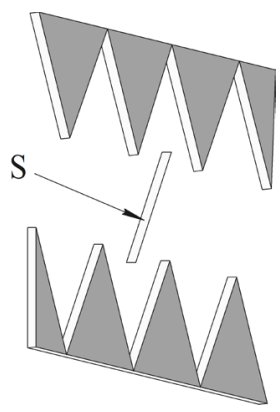

Fig. 4. To the calculation of the vector magnetic potential

To carry out variant calculations, it is necessary to determine the numerical values of the vector magnetic potential $A_{d e}$ at the boundary de (Fig. 2) for all 55 two-dimensional regions of the synthesized structures.

According to the results of $3 \mathrm{D}$ analysis, it was found that the value of the vector magnetic potential $A_{d e}$ depends on the filling of the working gap of the matrix with metal elements. So, the so-called filling factor $k_{m}$ was introduced for consideration, which connects the basic geometric relations $\left(X_{1}=b / a, X_{2}=\alpha\right)$ of the elements of the separator matrix,

$$
k_{m}=\frac{b}{a} \operatorname{ctg} \alpha
$$

For structural variants of the matrix of the electromagnetic separator, which were investigated, the fill factor $\mathrm{km}$ was, respectively: for variant $1-k_{m}=0.3532$; for option $2-k_{m}=0.616$; for option $3-k_{m}=0.769$.

Table 1 shows the calculated values of the fill factor $k_{m}$ with varying the geometric relationships $X_{1}=b / a$ and $X_{2}=\alpha$ in the ranges: $X_{1}=b / a=0.18 \ldots 0.4, X_{2}=\alpha=0.1 \pi \ldots 0.18 \pi$.

Based on the data obtained, a graph was constructed (Fig. 5), which describes the dependence of the vector magnetic potential $A_{d e}$ on the fill factor $k_{m}$. Using this graph, the $A_{d e}$ values are determined by interpolation for any intermediate points belonging to the range $0.3532 \leq k_{m} \leq 0.769$.

Table 1

Fill factor $k_{m}$

\begin{tabular}{cccccccc}
\hline$\alpha / \boldsymbol{0 . 1 8}$ & $\mathbf{0 . 2 0}$ & $\mathbf{0 . 2 5}$ & $\mathbf{0 . 2 8}$ & $\mathbf{0 . 3 0}$ & $\mathbf{0 . 3 5}$ & $\mathbf{0 . 4 0}$ \\
\hline $0.11 \pi$ & 0.495 & 0.549 & 0.687 & 0.769 & 0.824 & 0.962 & - \\
$0.12 \pi$ & 0.455 & 0.505 & 0.631 & 0.707 & 0.758 & 0.884 & - \\
$0.13 \pi$ & 0.416 & 0.462 & 0.578 & 0.647 & 0.693 & 0.809 & 0.924 \\
$0.14 \pi$ & 0.386 & 0.429 & 0.536 & 0.600 & 0.643 & 0.751 & 0.858 \\
$0.15 \pi$ & 0.3532 & 0.393 & 0.491 & 0.55 & 0.589 & 0.687 & 0.785 \\
$0.16 \pi$ & 0.325 & 0.361 & 0.451 & 0.505 & 0.541 & 0.631 & 0.722 \\
$0.17 \pi$ & 0.312 & 0.346 & 0.433 & 0.485 & 0.520 & 0.606 & 0.693 \\
$0.18 \pi$ & 0.277 & 0.308 & 0.385 & 0.431 & 0.462 & 0.539 & 0.616
\end{tabular}




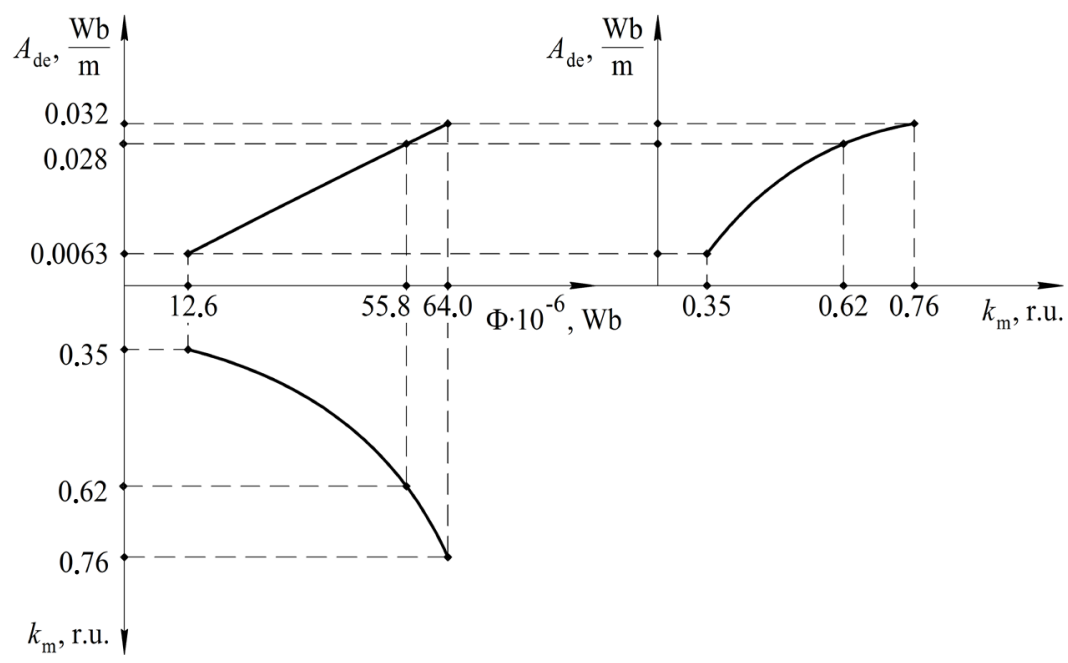

Fig. 5. Dependence of the vector magnetic potential $A_{d e}$ on the fill factor $k_{m}$

To calculate the $A_{d e}$ values at intermediate points of the considered interval $0.3532 \leq k_{m} \leq 0.769$, an approximating function (regression) was constructed using the least squares method [17]:

$$
A_{d e}=-0.1349 k_{m}^{2}+0.2132 k_{m}-0.0522 \text {. }
$$

Based on the results of polynomial approximation (2), the vector magnetic potential $A_{d e}$ was determined for various combinations of $X_{1}$ and $X_{2}$. For clarity, the results of calculating $A_{d e}$ are shown in the form of a volumetric graph (Fig. 6).

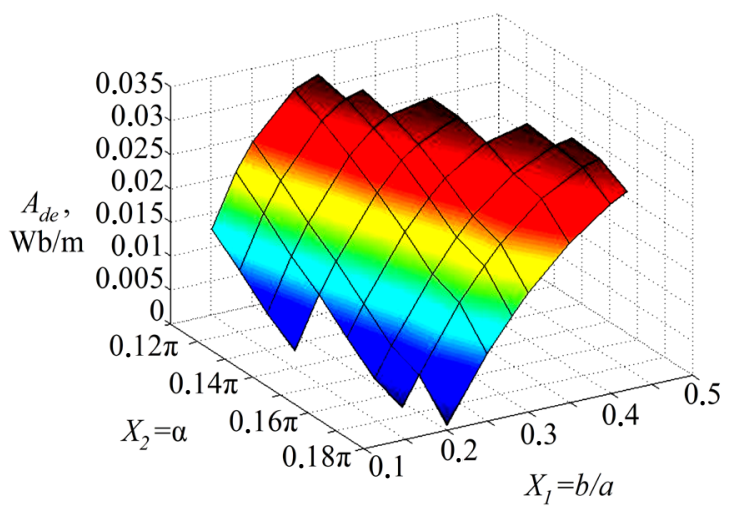

Fig. 6. Distribution of vector magnetic potential $A_{d e}$

Thus, the values of the vector magnetic potential $A_{d e}$ have been determined for various combinations of geometric similarity criteria $X_{1}$ and $X_{2}$ of the selected structure of the separator matrix.

These data were used to set the boundary conditions in a computational 2D experiment when choosing an electromagnetic separator matrix with rational geometric dimensions.

\section{Optional calculations of the magnetic matrix of an electromagnetic separator using two- dimensional models of working zones}

For the basic model of the separator (option 3), during the computational experiment, the fraction $P_{b}$ of the area of the working zone (pole-to-pole gap) of the matrix was determined, in which the value of the force parameter $f_{n p}$ satisfies the requirement:

$$
f_{\min } \leq f_{n p} \leq f_{\max },
$$


where $f_{\min }$ - the minimum threshold value of the power $f_{\min }=(H \operatorname{grad}(H))_{\min }$, at which, based on the experience of separator design, a sufficiently high efficiency of extraction of ferromagnetic inclusions is ensured (in the calculations, the value $f_{\min }=3 \cdot 10^{8} \mathrm{~A}^{2} / \mathrm{m}^{3}$ was taken); $f_{\max }$ - the maximum value of the specific reduced force, which for the base model was $f_{\max }=(H \operatorname{grad}(H))_{\max }=8.6 \cdot 10^{9} \mathrm{~A}^{2} / \mathrm{m}^{3}$.

This part of the area of the working area $P_{b}$ was expressed as a percentage in relation to the value $P$ of the entire area of the working area: $\gamma=P_{b} / P$. In this case, the parameter $\gamma$ depends significantly on the geometric relationships $X_{1}$ and $X_{2}$ and can be defined as the effective area of the working area of the matrix [14]. For the basic version of the separator, the parameter $\gamma$ was $\gamma=5.3 \%$. Some of the calculation results obtained during the computational experiment are shown in Fig. 7.

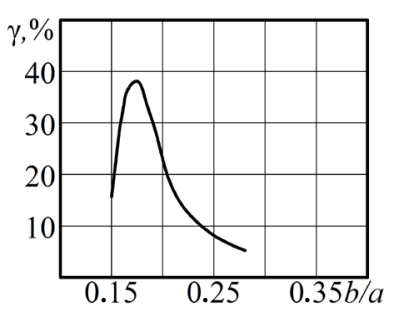

a

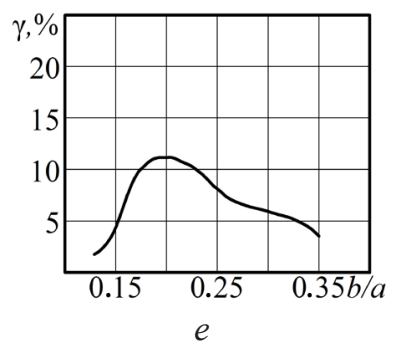

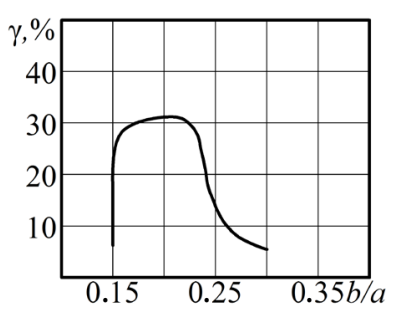

b

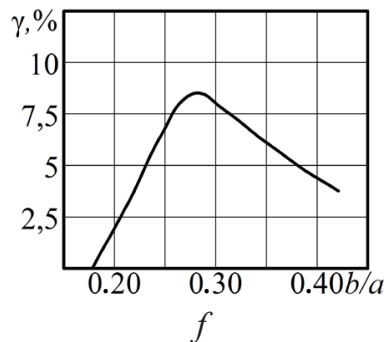

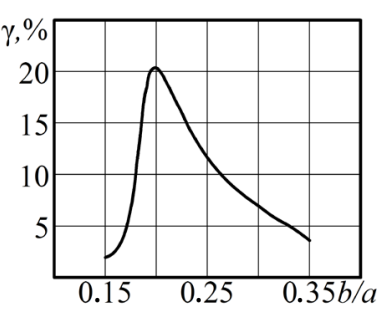

$c$

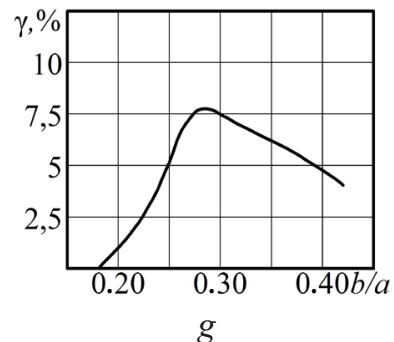

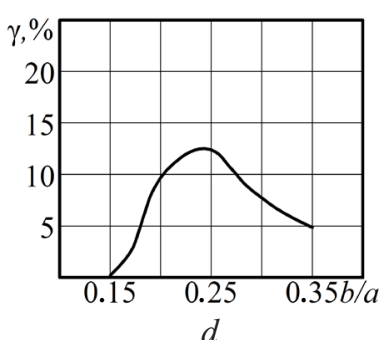

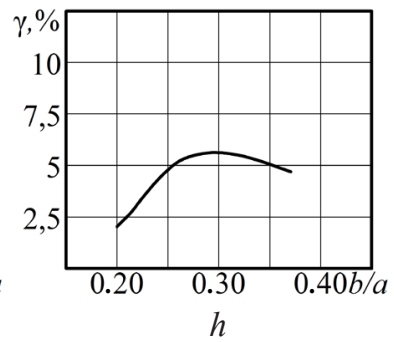

Fig. 7. Results of optional calculations of the parameter $\gamma$ : $a-X_{2}=\alpha=0.11 \pi ; b-X_{2}=\alpha=0.12 \pi ; c-X_{2}=\alpha=0.13 \pi ; d-X_{2}=\alpha=0.14 \pi ; e-X_{2}=\alpha=0.15 \pi ;$ $f-X_{2}=\alpha=0.16 \pi ; g-X_{2}=\alpha=0.17 \pi ; h-X_{2}=\alpha=0.18 \pi$

Options of the investigated systems that satisfy the condition:

$$
\gamma\left(X_{1}, X_{2}\right) \rightarrow \max
$$

The calculation results obtained during the computational experiment showed that condition (4) is satisfied by the structure of the matrix with geometric similarity criteria $X_{1}=b / a=0.18$; $X_{2}=\alpha=0.11 \pi$, for which the parameter $\gamma$ takes the maximum value $\gamma=36.5 \%$. For comparison, the $\gamma$ parameter for the basic structure was $5.3 \%$. Thus, the following parameters should be considered rational: $X_{1}=b / a=0.18$ i $X_{2}=\alpha=0.11 \pi$.

The reliability of the results obtained during the computational experiment was confirmed by comparison with the results obtained by other researchers [2, 18-20]. According to estimated calculations, the productivity of an electromagnetic separator with rational geometric parameters of a plate matrix will increase by 3-5\% with a constant extraction efficiency of ferromagnetic inclusions in comparison with the basic version of the device.

\section{Discussion of the results of studying the distribution of the magnetic field in the matrix of the polygradient separator}

The paper substantiates an approach to multivariate calculations of the magnetic field distribution, based on the use of the advantages of two- and three-dimensional computer modeling. This made it possible to reduce the time costs associated with three-dimensional modeling, and thus increase the computational efficiency of calculations in general.

The practical implementation of the proposed approach provided for the use of a limited number of three-dimensional models of the electromagnetic system of the separator, as auxiliary 
ones, for finding the boundary conditions of the computational domains. Three versions of the electromagnetic separator were chosen with different values of the working air gap $\delta$ : minimum $\delta_{\min }$, maximum $\delta_{\max }$ and average $\delta_{a v}\left(\delta_{\min } \leq \delta_{a v} \leq \delta_{\max }\right)$. Fig. 3 shows, for example, a three-dimensional geometric model of an electromagnetic separator (Fig. 3, a) and a picture of the magnetic field distribution (Fig. 3, b) for a variant of the device with the maximum value of the working gap $\left(\delta_{\max }=53 \mathrm{~mm}\right)$.

To solve the second problem of the study, the boundary conditions were determined, in particular the vector magnetic potential $A_{d e}$ in the section $d e$ (Fig. 2) for the working areas of the matrix using the selected three-dimensional models of the electromagnetic separator. An approximating regression model is built - formula (2), with the help of which the vector magnetic potential $A_{d e}$ is determined for various combinations of geometric similarity criteria $X_{1}$ and $X_{2}$.

The boundary conditions for the vector magnetic potential $A_{d e}$, obtained from the results of three-dimensional computer modeling, were further used to carry out variant calculations and determine the rational geometric parameters of the plate elements of the magnetic matrix according to the criterion $\gamma$ of the effective area of the working area of the matrix using two-dimensional models. It is shown that the variants of the systems under study that satisfy condition (4) will be considered rational. The analysis of the results obtained, presented in Fig. 7, made it possible to determine the numerical values of the geometric similarity criteria $\left(X_{1}=b / a=0.18 ; X_{2}=\alpha=0.11 \pi\right)$ for which the parameter $\gamma$ takes the maximum value $(\gamma=36.5 \%)$.

At the initial stage of the study of the spatial distribution of the magnetic field in the working gaps of the matrix of the polygradient separator, it was assumed to use the numerical finite element method in a three-dimensional formulation. The undoubted advantage of 3D modeling is the ability to calculate the magnetic field in any structural element of the electromagnetic separator and to approximate the calculation results to real processes. At the same time, the analysis of the field distribution for 55 computer 3D models of the electromagnetic separator, which differ in the geometric dimensions of the matrix elements, required a significant computation time. Taking this into account, a reasonable approach to carrying out multivariate calculations of the magnetic field distribution, based on the use of the advantages of two- and three-dimensional computer modeling. This made it possible to reduce the time costs associated with three-dimensional modeling, and thus increase the computational efficiency of calculations in general.

The distribution of the magnetic field in the working areas of the matrix is also influenced by other factors, for example, the shape of the matrix elements. The work investigated plate matrices, the elements of which have the shape of an equilateral triangle. Determination of the influence on the distribution of the magnetic field of plate elements with a different geometric shape, for example, trapezoidal, was not included in the research tasks. The study of this influence can be the subject of further research work.

\section{Conclusions}

1. A combined approach to the analysis of magnetic fields is substantiated, taking into account the advantages of finite element analysis in both two-dimensional and three-dimensional formulations. This approach is capable of providing the required computational efficiency and accuracy.

2. An approach is proposed to determine the boundary condition (vector magnetic potential) at the boundary de of two-dimensional computational domains, based on the study of the spatial distribution of the magnetic field in the working gap of the electromagnetic separator. The numerical values of the vector magnetic potential at the boundary of the region are obtained for three three-dimensional models of the electromagnetic system of the separator using the Magnet module of the Infolytica software package. The calculation of the boundary conditions for all other models was carried out by interpolation.

3. The choice of a rational version of the polygradient matrix of the electromagnetic separator is carried out according to the criterion $\gamma$ of the effective area of the working area. The analysis carried out using the method of simple enumeration of options showed that the structure with the parameters $X_{1}=b / a=0.18$ and $X_{2}=\alpha=0.11 \pi$ in terms of a high value of the effective area $\gamma$ of the 
working area $(\gamma=36.5 \%)$ can be considered rational. A variant of the structure of the polygradient matrix with rational parameters $X_{1}=b / a=0.18$ and $X_{2}=\alpha=0.11 \pi$ is adopted in comparison with the basic design of a separator with parameters $X_{1}=b / a=0.28, X_{2}=\alpha=0.11 \pi$, chosen empirically, is characterized by the following:

- the maximum intensity $H_{\max }$ and the maximum intensity gradient $\operatorname{grad}(H)_{\max }$ of the magnetic field of the structure with rational parameters exceed almost 3 times the corresponding indicators of the basic structure;

- for a structure with rational parameters, the value of the effective area of the working zone $\gamma$ is almost 7 times larger than that of the base one.

\section{Acknowledgments}

The authors express their sincere gratitude to the staff of the Electromechanical Systems Department of the Institute of Electrodynamics of the National Academy of Sciences of Ukraine for their help in carrying out numerical calculations using the Infolytica software package.

\section{References}

[1] Soloshych, I., Shvedchykova, I. (2016). Development of systematics ranked structure of environmental protecting equipment for cleaning of gas emissions, wastewater and solid waste. Eastern-European Journal of Enterprise Technologies, 6 (10 (84)), 17-23. doi: https://doi.org/10.15587/1729-4061.2016.86462

[2] Svoboda, J. (2004). Magnetic techniques for the treatment of materials. Springer, 642. doi: https://oi.org/10.1007/ $1-4020-2107-0$

[3] Ge, W., Encinas, A., Araujo, E., Song, S. (2017). Magnetic matrices used in high gradient magnetic separation (HGMS): A review. Results in Physics, 7, 4278-4286. doi: https://doi.org/10.1016/j.rinp.2017.10.055

[4] Li, W., Han, Y., Xu, R., Gong, E. (2018). A Preliminary Investigation into Separating Performance and Magnetic Field Characteristic Analysis Based on a Novel Matrix. Minerals, 8 (3), 94. doi: https://doi.org/10.3390/min8030094

[5] Tolmachev, S. T., Bondarevskyi, S. L., Il'chenko, A. V. (2020). Magnetic properties of multicomponent heterogeneous media with a doubly periodic structure. Electrical Engineering \& Electromechanics, 1, 29-38. doi: https://doi.org/10.20998/ 2074-272x.2020.1.05

[6] Zeng, J., Tong, X., Yi, F., Chen, L. (2019). Selective Capture of Magnetic Wires to Particles in High Gradient Magnetic Separation. Minerals, 9 (9), 509. doi: https://doi.org/10.3390/min9090509

[7] Chen, H., Bockenfeld, D., Rempfer, D., Kaminski, M. D., Liu, X., Rosengart, A. J. (2008). Preliminary 3-D analysis of a high gradient magnetic separator for biomedical applications. Journal of Magnetism and Magnetic Materials, 320 (3-4), $279-284$. doi: https://doi.org/10.1016/j.jmmm.2007.06.001

[8] Milykh, V. I. (2018). The system of automated formation of electrical machines computational models for the femm software environment. Tekhnichna Elektrodynamika, 2018 (4), 74-78. doi: https://doi.org/10.15407/techned2018.04.074

[9] Milykh, V. I. (2018). Numerically-field analysis of the adequacy of the design data of three-phase induction motors and the method of their refinement on this basis. Tekhnichna Elektrodynamika, 2018 (1), 47-55. doi: https://oi.org/10.15407/ techned2018.01.047

[10] Grebenikov, V. V., Gamaliia, R. V. (2019). Comparative Analysis of Two Types of Generators with Permanent Magnets for Wind Turbine. 2019 IEEE International Conference on Modern Electrical and Energy Systems (MEES). doi: https:// doi.org/10.1109/mees.2019.8896375

[11] Dobzhanskyi, O., Hossain, E., Amiri, E., Gouws, R., Grebenikov, V., Mazurenko, L. et. al. (2019). Axial-Flux PM Disk Generator With Magnetic Gear for Oceanic Wave Energy Harvesting. IEEE Access, 7, 44813-44822. doi: https://doi.org/ 10.1109/access.2019.2908348

[12] Shvedchykova, I., Lutsenko, I., Romanchenk, J. (2015). A study ofpolygradient media structure regularities. Eastern-European Journal of Enterprise Technologies, 4 (7 (76)), 62-67. doi: https://doi.org/10.15587/1729-4061.2015.47785

[13] Shvedchykova, I., Romanchenko, J., Nikitchenko, I. (2017). Comparative analysis of inhomogeneity degree of magnetic field of polygradient magnetic separators for purification of bulk materials. 2017 International Conference on Modern Electrical and Energy Systems (MEES). doi: https://doi.org/10.1109/mees.2017.8248873

[14] Gerlici, J., Shvedchikova, I. A., Romanchenko, J. A., Nikitchenko, I. V. (2018). Determination of the rational geometrical parameters of plate type elements of magnetic matrix of the polygradient separator. Electrical Engineering \& Electromechanics, 4, 58-62. doi: https://doi.org/10.20998/2074-272x.2018.4.10

[15] Maliar, V. S. (2018). Teoretychni osnovy elektrotekhniky. Lviv: Vydavnytstvo Lvivskoi politekhniky, 416. 
[16] Simcenter MAGNET. Available at: https://www.plm.automation.siemens.com/global/en/products/simcenter/magnet.html

[17] Chekotovskyi, E. (2018). Statystychni metody na osnovi Microsoft Excel 2016. Kyiv: Znannia, 408.

[18] Ren, L., Zeng, S., Zhang, Y. (2015). Magnetic field characteristics analysis of a single assembled magnetic medium using ANSYS software. International Journal of Mining Science and Technology, 25 (3), 479-487. doi: https://oi.org/10.1016/ j.ijmst.2015.03.024

[19] Song, C., Ning, G., Yuan, Z., Jing, L., Hui, C., Yao, M. (2014). Investigation of the influence of different matrix rotation angles on the surrounding magnetic field in a uniform magnetic field. Ming Metall Eng, 34, 290-294.

[20] Shun, Z., Liang, S., Juan, W., Yue, W. (2012). Experimental study on magnetic separation by conical flux gathering media and optimization of its cone angle. Min Process Equip, 74-79.

Received date 15.12.2020

(C) The Author(s) 2021

Accepted date 12.03.2021

This is an open access article under the CC BY license

Published date 13.09.2021

(http://creativecommons.org/licenses/by/4.0).

How to cite: Jasim, J. M. J., Shvedchykova, I., Panasiuk, I., Romanchenko, J., Melkonova, I. (2021). Increasing the efficiency of multy-variant calculations of electromagnetic field distribution in matrix of a polygradient separator. EUREKA: Physics and Engineering, 5, 69-79. doi: https://doi.org/10.21303/2461-4262.2021.001713 